\title{
POTENTIAL FIELD EXTRAPOLATION USING THREE COMPONENTS OF SOLAR VECTOR MAGNETOGRAM WITH A FINITE FIELD OF VIEW
}

\author{
G. ALLEN GARY \\ Space Sciences Laboratory/ES82, George C. Marshall Space Flight Center/NASA, Huntsville, AL 35812, U.S.A. ${ }^{1}$
}

\begin{abstract}
The potential magnetic field from a finite planar boundary is extrapolated into the upper hemisphere using all three of the magnetic field components. The method determines, first, the transverse field assoicated with the observed normal magnetic intensity, and, then by subtraction, the method determines the associated transverse magnetic field observed in the interior (i.e., in the field of view) of the magnetogram which is due to the normal flux exterior to the field of view of the magnetograph. The combination of the observed normal flux of the interior and the approximation of the exterior normal flux is employed to calculate the potential field. The formulation of the problem results in an ill-posed integral inversion problem which is solved using the singular value decomposition (SVD) techniques in conjunction with an appropriate Tikhonov-Phillips filter. The use of the technique is in correcting potential field calculations which are influence by out of view fluxes, e.g. for a high spatial resolution vector magnetogram with a small field of view in which there is no supporting data exterior. Such a corrected potential field is needed for reference when specifying the nonpotentiality of a region (i.e., magnetic shear studies). The problem studied is also important in providing a regularized solution of the Cauchy potential problem. The methods provides a much larger range of convergence than the method of Gary and Musielak (1992), and, in fact, is stable in the total upper hemisphere.
\end{abstract}

\section{Introduction}

The magnetic field extrapolation method derived herein uses all three components of a vector magnetogram within a relative small, finite field of view to determine a corrected potential magnetic field over the field of view as influenced by the exterior flux. In the standard potential methods where one uses only the normal component, a problem arises if, either the normal flux from an active region is not entirely captured by the magnetogram because of the restricted field of view of the magnetograph, or one or more additional active regions outside the field of view of the magnetogram effect the potential field configuration of the selected active region. This is clearly seen in the soft x-ray images from Yohkoh. If only the normal magnetic field within field of view of a magnetogram is used in a standard integral or Fourier potential method, the method will give a false potential field since all the important surface field is not taken in to account. In order to try to correct this error, the procedure we describe below uses the additional information contained in the transverse field within the magnetogram to reconstruct a representative field for the normal component outside of the magnetogram. Hence this extension of the normal field can be used in an integral potential method to calculated the magnetic field which includes the effects of both the normal field resolved by the magnetogram and a reconstructed magnetic field outside of the magnetogram. The basic idea is that the magnetic field outside the magnetogram field of view produces some effect on the transverse field of the magnetogram. Then by using the transverse field, the normal magnetic field distribution outside the magnetogram can estimated.

There are many ways to extrapolate the solar magnetic field. The place of this potential method in terms of the various schemes to extrapolate the magnetic field is shown in Figure 1. This method uses all three components of the observed magnetic field to arrive a potential solution. General magnetic fields are characterized by being potential or current carrying. The potential methods are separated into planar and spherical models. The first

${ }^{1}$ E-mail: Gary@SSL.MSFC.NASA.GOV 
representation of a solar magnetic potential field was presented by Schmidt $(1964,1965,1966)$ and used by Harvey (1966). This solution was for a planar boundary with Bz given on the plane. Semel (1967) allowed the inclined line-of-sight observations to be employed. These programs were integral solutions; an eigenfunction approach was developed by Teuber (1982) which used fast Fourier transforms. The unique method described here extends the planar representation by using the full content of the vector magnetogram.

The spherical geometry case has a long history, starting with Gauss' spherical harmonic analysis of the earth's magnetic field. Altschuler and Newkirk was the first to developed an eigenfunction spherical potential model for the whole disk with an upper source surface. A finite-difference approach was developed by Adams and Pneuman (1976). A much faster code was developed by Riesebiedder and Neubauer (1979) using specific orthogonality relations. A formulation by Levine et al. (1982) employed a non-spherical source surface. These methods used integrated observations of $\mathrm{Bl}$ over an entire solar rotation. Observations using instanteous observations were developed. Sakurai (1982) developed a Green's function approach whcih had the source surface at infinity. Kopp and Poletto (1990) using eigenfunction approach developed a code with a finite-variable distance source surface. The vector magnetograms could be used to extend to spherical representations for finite field of views, but only the planar case is given herein.

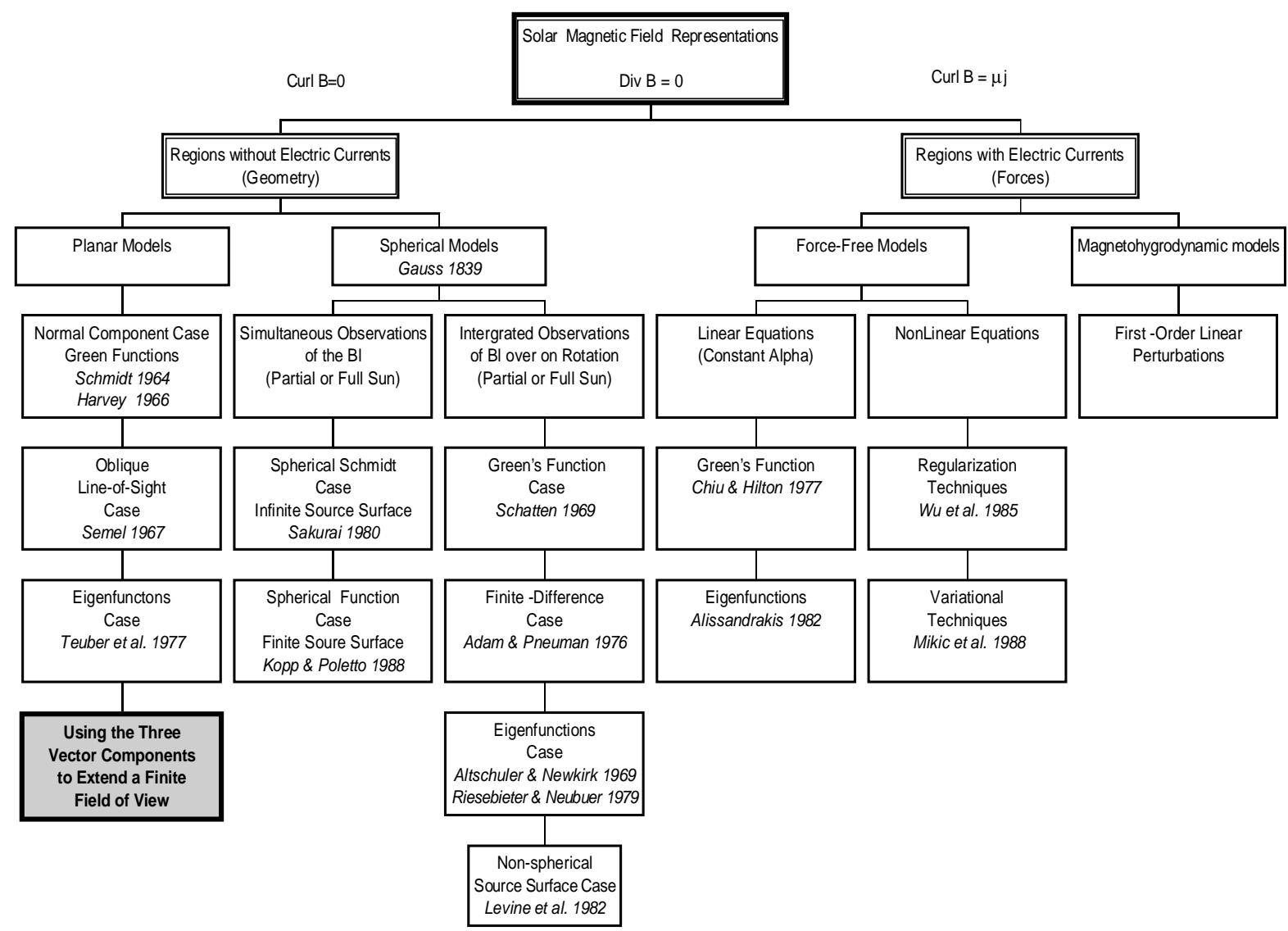

Figure 1. Solar Magnetic Field Extrapolation Methods. The divisions of the various magnetic field representations are shown. The main division is between the potential and current carrying fields. The potential field are subdivided geometrically into planar and spherical boundary conditions. The regions with current are subdivided inot force-free and non-force-free regions. The extrapolation method using all three components, discussed herein, in shown in relation to the other methods. Its uniqueness is in extending the field of view by employing the transverse components to estimate the out of view flux. 
For regions which carried currents, in which on has a linear force-free field condition, Nakagawa et al. (1977), Chiu and Hilton (1981), and Alissandrakis (1982) developed Green's function and eigenfunctions solutions. For nonlinear fields the problem is much harder (Gary 1990). Mikic et al. (1988m 1989, 1990), Wu et al. (1990), and Roumeliotis (1994) have developed approximate methods to handle the nonlinear force-free field case. For current-carrying fields the transverse components are used either to determined the linear constatnt $\alpha$ by a least square solution (Kress 1897) or are needed to extrapolate the field; and the formulation given here is obviously not appropriate for the current carrying filelds. However, potential field theory still remains an important part of the analysis of the solar magnetic fields, (e.g. in shear analysis) and the corrections given herein can be important in given the potential field. Furthermore, the regularization schemes can be carried into the nonlinear regime (Morozov 1993).
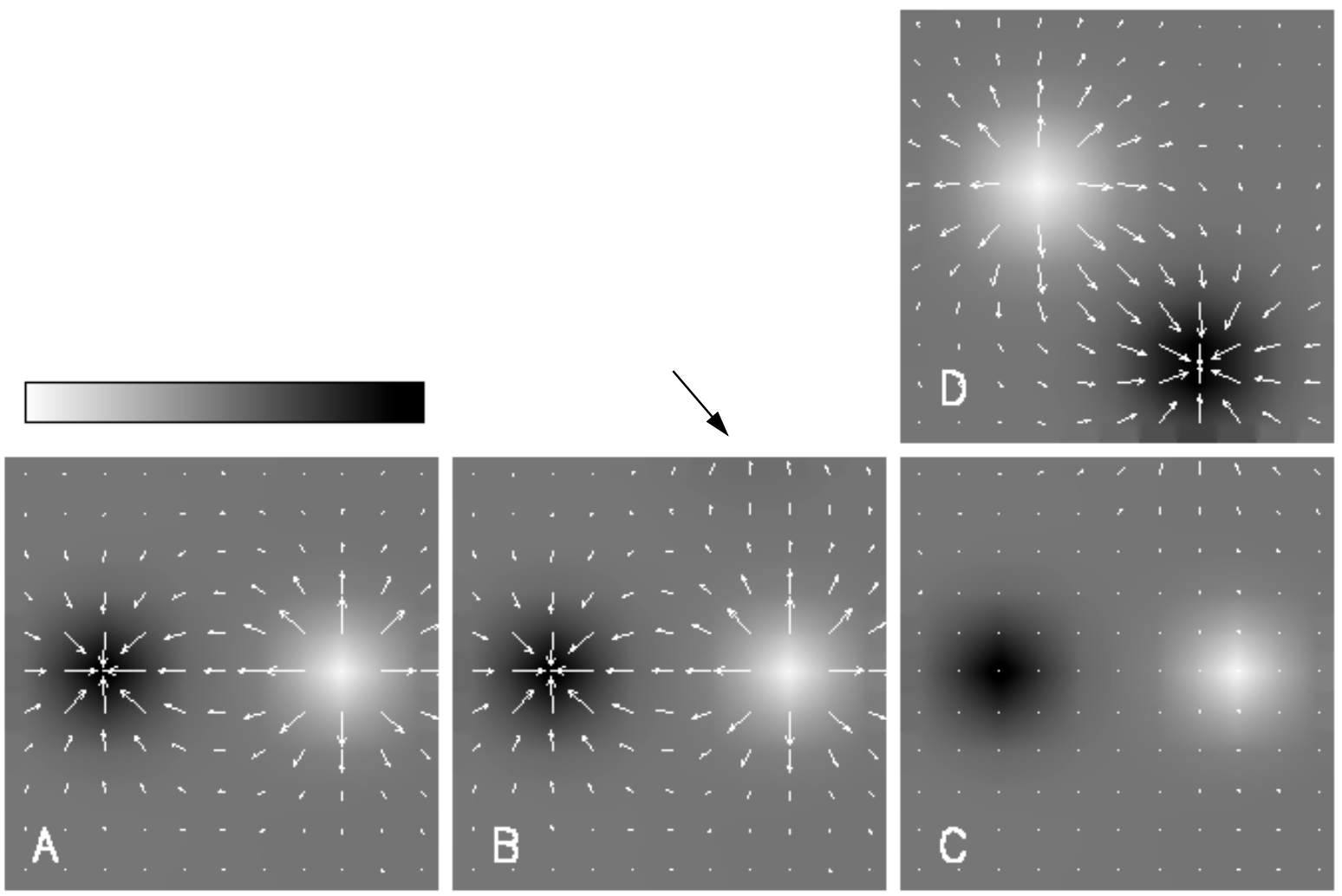

Figure 2. Illustration of the effect of out of view flux contributions. (A) A double dipole configuraton is seen in the field of view of the magnetogram. The normal magnetic field intensity is seen as gray levels and the transverse field is shown as arrows. (B) The same configuration as in panel A but with an additonal dipole configuration outside the field of view (as given by D) with the effect of the exterior configurations being apparent at the edge of the field of view (black arrow). (C) The transverse field of configuration $A$ is subtracted from transverse field of configuration $B$ to produce the transverse field of the exterior field as seen in the magnetogram. (D) The exterior field of view, unseen by the magnetogram, is shown. Its contribution in the interior field of view is just that given by panel $C$.

An illustrated effect of the influence of external flux on the transverse field within field of view of the magnetogram is given in Figure 2. The panel A, B, and C are in the field of view of the magnetogram whereas 
panel D is exterior to the field of view of the magnetogram. Panel A is a synthetic magnetogram of two dipoles with no external dipoles outside the field of view. In panel B the effects of two additional exterior dipoles are seen where the transverse field above the positive (light) region in B is influenced by the effect of the negative field outside the field of view (i.e. panel D). Panel A is the potential solution of panel $B$ given the flux seen only in panel B. The effect the exterior field can be determine by subtracting the transverse filed generated by the interior field (i.e. the potential field) from the observed transverse field. This result is given by panel C. The main point of this paper is to use the field in panel $\mathrm{C}$ to estimate the flux as given in panel D for the potential case of a planar boundary.

A motivation of this approach is that the potential field gives the three components of the field, $B_{x}, B_{y}, B_{z}$, from one scalar function $\phi$. Hence the $B_{x}$ component is given by

$$
B_{x}=\int \frac{\partial \phi}{\partial n} \frac{1}{r} d a=\int B_{z} \frac{1}{r} d a
$$

Hence, given $B_{x}$ we can solve for $B_{z}$ in a classical integral inversion problem. This is what we set out in this paper to specifically show by using a regularization scheme to handle the ill-posedness of the problem. The illposedness is the classical Fredholm integral equation of the first kind (Craig and Brwon 1986).

In $\S 2$ the general Biot-Savart law is given for the magnetostatic problem and in $\S 3$ the formulation of the inverse problem is layed out. In $\S 4$ the numerical problem is formulated with specific numerical examples given in $\S 5$. In the conclusion (§6) we discuss the theory relevancy to the Cauchy problem.

\section{Extended Biot-Savart Law}

We will use the extended Biot-Savart law giving the magnetic field within a volume V bounded by the surface S. This formula is derived by starting from the vector form of Green's second identity,

$$
\int_{\mathrm{V}}(\mathbf{Q} \cdot \nabla \times \nabla \times \mathbf{A}-\mathbf{A} \cdot \nabla \times \nabla \times \mathbf{Q}) \mathrm{dv}^{\prime}=\int_{\mathrm{S}}(\mathrm{A} \times \nabla \times \mathbf{Q}-\mathbf{Q} \times \nabla \times \mathbf{A}) \cdot \mathbf{n} \mathrm{da} \mathbf{a}^{\prime}
$$

and letting $\mathbf{A}$ be the magnetic vector potential and $\mathbf{Q}$ be the appropriate Green's function, i.e.

$$
\mathbf{B}=\nabla \times \mathbf{A}, \quad \text { and } \quad \mathbf{Q}=\nabla(1 / \mathrm{r}) \times \mathbf{a},
$$

where $\mathbf{a}$ is an arbitrary, but fixed, unit vector and $\mathbf{r}=\left|\mathbf{x}-\mathbf{x} \mathbf{x}^{\prime}\right|$ is the distance between the field point and the source point. The magnetic field anywhere in the volume, $\mathbf{B}(\mathbf{x})$, is given explicitly by the electric current density within the volume, $\mathbf{J}\left(\mathbf{x}^{\prime}\right)$, and the magnetic field components on the boundary surface, $\mathbf{n} \cdot \mathbf{B}\left(\mathbf{x}^{\prime}\right)$ and $\mathbf{n} \times \mathbf{B}\left(\mathbf{x}^{\prime}\right)$, which receives addition contribution from the electric currents on the surface and exterior to the volume. The extended Biot-Savart law is given by the following formula (Stratton $1941 \mathrm{sec} .4 .14$ ):

$$
\mathbf{B}(\mathbf{x})=\mu / 4 \pi \int_{\mathrm{V}} \mathbf{J}\left(\mathbf{x}^{\prime}\right) \times \nabla^{\prime}(1 / \mathrm{r}) \mathrm{dv^{ \prime }}-1 / 4 \pi \int_{\mathrm{S}}\left(\mathbf{n} \times \mathbf{B}\left(\mathbf{x}^{\prime}\right)\right) \times \nabla^{\prime}(1 / \mathrm{r}) \mathrm{da} \mathrm{a}^{\prime}-1 / 4 \pi \int_{\mathrm{S}}\left(\mathbf{n} \cdot \mathbf{B}\left(\mathbf{x}^{\prime}\right)\right) \nabla^{\prime}(1 / \mathrm{r}) \mathrm{da} .
$$

In the appendix an alternate derivation of this equation is given. We note that the magnetic field intensity components used in the surface integrals are not the potential field solution and the field in the volume is not even force-free since $\mathbf{J} \neq 0$.

\section{Formulation of the Inversion Integral}

In this section, we consider the flux contribution outside the magnetogram and show how these contributions can be used for the potential case. In the standard methods, the assumption is that $\mathrm{B}_{\mathrm{z}}=0$ outside the field of view (or periodicity). In this section an inversion integral is derived to give an estimate of the flux outside of the field of view. Now, for the case of only subsurface electric currents, 
the volume integral is zero and the two surface integrals are equal; hence, for the potential field, the equation becomes simply,

$$
\mathbf{B}(\mathbf{x})=-1 / 2 \pi \int_{\mathrm{S}}\left(\mathbf{n} \cdot \mathbf{B}\left(\mathbf{x}^{\prime}\right)\right) \nabla^{\prime}(1 / \mathrm{r}) \mathrm{da},
$$

which was used by H. U.Schmidt (1963) for solar magnetic field extrapolation. Now if we consider the problem of the upper half plane, with the contribution from the covering spherical shell being zero, i.e. conserved flux, then the last equation can be written as an integral over the region of the magnetogram $M$ and an integral over the region outside of $M$, called $\Gamma M$, where the plane $\mathrm{z}=0$ is $\Gamma$ :

$$
\mathbf{B}(\mathbf{x})=-1 / 2 \pi \int_{M}\left(\mathbf{n} \cdot \mathbf{B}\left(\mathbf{x}^{\prime}\right)\right) \nabla^{\prime}(1 / \mathrm{r}) \mathrm{da} \mathrm{a}^{\prime}-1 / 2 \pi \int_{\Gamma^{\mathrm{M}} \mathrm{M}}\left(\mathbf{n} \cdot \mathbf{B}\left(\mathbf{x}^{\prime}\right)\right) \nabla^{\prime}(1 / \mathrm{r}) \mathrm{da}
$$

We now rearrange the equation in terms of the unknown contribution from the region $I \mathrm{LM}$, i.e. outside the field of view of the magnetogram, and write the normal and transverse components out explicitly. The normal component is

$$
\mathbf{n} \cdot 1 / 2 \pi \int_{\mathrm{M}}\left(\mathbf{n} \cdot \mathbf{B}\left(\mathbf{x}^{\prime}\right)\right) \nabla^{\prime}(1 / \mathrm{r}) \mathrm{da} \mathrm{a}^{\prime}=-\mathbf{n} \cdot 1 / 2 \pi \int_{\Gamma \mid M}\left(\mathbf{n} \cdot \mathbf{B}\left(\mathbf{x}^{\prime}\right)\right) \nabla^{\prime}(1 / \mathrm{r}) \mathrm{da} \mathrm{a}^{\prime}-\mathbf{n} \cdot \mathbf{B}(\mathbf{x})
$$

and, more importantly, the transverse component is

$$
\mathbf{n} \times 1 / 2 \pi \int_{\Gamma \mid M}\left(\mathbf{n} \cdot \mathbf{B}\left(\mathbf{x}^{\prime}\right)\right) \nabla^{\prime}(1 / \mathrm{r}) \mathrm{da}^{\prime}=-\mathbf{n} \times 1 / 2 \pi \int_{\mathrm{M}}\left(\mathbf{n} \cdot \mathbf{B}\left(\mathbf{x}^{\prime}\right)\right) \nabla^{\prime}(1 / \mathbf{r}) \mathrm{da} \mathbf{a}^{\prime}-\mathbf{n} \times \mathbf{B}(\mathbf{x})
$$

Now if we evaluate the above equations with $\mathbf{x}$ on the surface in $M$, i.e. within the field of view. Then the normal compoent integral gives us nothing new since the left hand side of the equation is zero. This comes from the identity ${ }^{2}, \mathbf{n} \cdot \nabla^{\prime}(1 / r)=0$ for $x^{\prime}=\Gamma$ where $z=z^{\prime}=0$ and $r \neq 0$; and for a spherical cap $C$ about the singularity point

$$
\text { - } \mathbf{n} \cdot 1 / 2 \pi \int_{\mathrm{C}}\left(\mathbf{n} \cdot \mathbf{B}\left(\mathbf{x}^{\prime}\right)\right) \nabla^{\prime}(1 / \mathrm{r}) \mathrm{da} \mathbf{a}^{\prime}-\mathbf{n} \cdot \mathbf{B}(\mathbf{x}) \equiv \mathbf{0} .
$$

The transverse integral equation above, however, provides us the additional information, for $\mathbf{x} \in M$, it states that part of the transverse field in the magnetogram $\mathbf{x} \in M$ is produced by the flux outside in $I \mathrm{M} M$ and this is equal to difference of the transverse field produced from only the field in $M$ and the observed transverse field in the magnetogram, $M$. Hence we have an integral equation for the normal component of the field outside of the magnetogram, i.e. for $\mathbf{n} \cdot \mathbf{B}(\mathbf{x})$ for $\mathbf{x} \in I M$, see per Figure 1.

Hence obtaining the normal component for the entire plane we can the obtained the potential field which accounts for the magnetic flux outside of the magnetogram. Writing the transverse integral equation out into its component form, we have:

\footnotetext{
${ }^{2}$ Note that for a small region about the point $\mathrm{z}=\mathrm{z}^{\prime}=0$ and $\mathrm{r}=0$ where there is the singularity, we can write

$$
\int_{V} \nabla^{2}\left(\frac{1}{r}\right) d v=\int_{V} \nabla \cdot\left(\nabla\left(\frac{1}{r}\right)\right) d v=\int_{a} \mathbf{n} \cdot \nabla\left(\frac{1}{r}\right) d a \int_{\Omega} \frac{\partial}{\partial r}\left(\frac{1}{r}\right) r^{2} d \Omega=-4 \pi
$$

which produces the identity.
} 


$$
\begin{aligned}
& \frac{1}{2 \pi} \iint_{\Gamma \backslash M} \frac{x-x^{\prime}}{\left(\left(x-x^{\prime}\right)^{2}+\left(y-y^{\prime}\right)^{2}\right)^{3 / 2}} B_{z}\left(x^{\prime}, y^{\prime}\right) d x^{\prime} d y^{\prime} \\
& =\frac{1}{2 \pi} \iint_{M} \frac{x-x}{\left(\left(x-x^{\prime}\right)^{2}+\left(y-y^{\prime}\right)^{2}\right)^{3 / 2}} B_{z}\left(x^{\prime}, y^{\prime}\right) d x^{\prime} d y^{\prime}-B_{x}(x, y)
\end{aligned}
$$

and

$$
\begin{aligned}
& \frac{1}{2 \pi} \iint_{\Gamma \backslash M} \frac{y-y^{\prime}}{\left(\left(x-x^{\prime}\right)^{2}+\left(y-y^{\prime}\right)^{2}\right)^{3 / 2}} B_{z}\left(x^{\prime}, y^{\prime}\right) d x^{\prime} d y^{\prime} \\
& =\frac{1}{2 \pi} \iint_{M} \frac{y-y^{\prime}}{\left(\left(x-x^{\prime}\right)^{2}+\left(y-y^{\prime}\right)^{2}\right)^{3 / 2}} B_{z}\left(x^{\prime}, y^{\prime}\right) d x^{\prime} d y^{\prime}-B_{y}(x, y)
\end{aligned}
$$

where $z=z^{\prime}=0$. Then we have a pair of integral equations that we must solve for $B_{z}\left(x^{\prime}, y^{\prime}\right)$ for $\left(x^{\prime}, y^{\prime}\right)$ outside of the magnetogram field of view, i.e. $\left(\mathrm{x}^{\prime}, \mathrm{y}^{\prime}\right) \in \Gamma M$. Inverting either of the last two equations are theoretical equivalent in term of solving for $\mathrm{Bz}$, but we will carry both equations by using complex notation. Multiplying the second equation by $\mathrm{l}$ and summing the equations, we have

$$
\begin{aligned}
& \frac{1}{2 \pi} \iint_{\Gamma \backslash M} \frac{\left(x-x^{\prime}\right) \pm \imath\left(y-y^{\prime}\right)}{\left(\left(x-x^{\prime}\right)^{2}+\left(y-y^{\prime}\right)^{2}\right)^{3 / 2}} B_{z}\left(x^{\prime}, y^{\prime}\right) d x^{\prime} d y^{\prime} \\
& =\frac{-1}{2 \pi} \iint_{M} \frac{\left(x-x^{\prime}\right) \pm \imath\left(y-y^{\prime}\right)}{\left(\left(x-x^{\prime}\right)^{2}+\left(y-y^{\prime}\right)^{2}\right)^{3 / 2}} B_{z}\left(x^{\prime}, y^{\prime}\right) d x^{\prime} d y^{\prime}-\left(B_{x}(x, y) \pm \imath B_{y}(x, y)\right),
\end{aligned}
$$

where the right hand side of the equation is defined to be $F(x, y)$ and is known from the direct observation of the vector magnetograph. This equation has the form of Fredholm integral equation of the first kind:

$$
\int_{c}^{d} \int_{a}^{b} K\left(x, y, x^{\prime}, y^{\prime}\right) B_{z}\left(x, y^{\prime}\right) d x^{\prime} d y^{\prime}=\quad F(x, y)
$$

where we wish to determine $\mathrm{B}_{\mathrm{z}}$. However, we note that $\left(\mathrm{x}^{\prime}, \mathrm{y}^{\prime}\right) \in(\mathrm{a}, \mathrm{b}) \in I \mathbb{} M$ but $(\mathrm{x}, \mathrm{y}) \in \mathrm{M}$. The inversion of this integral equation is a classical ill-posed problem (e.g., Glasko, 1984, Groetsch 1984, Craig and Brown 1986). However, various schemes have been developed to stablized the inversion. In the next section, the method of regularization is applied to the corresponding set of equations.

\section{Numerical Inversion Technique}

Numerical calculation of the integrals are obtained by integrating over individual, small grid cells, in which the magnetic field does not vary significantly, and summing. The average of the field within the grid cell is $\bar{B}_{z}$. In the numerical equations, the following notation is used for the $\mathrm{i}^{\text {th }}$ grid cell: $\xi=\mathrm{x}-\mathrm{x}$, and $\eta=\mathrm{y}-\mathrm{y}^{\prime}$. The integrals over $M$ and $I \mathrm{~W}$ and over the appropriate grid cells defined by $\left(\xi_{1}, \xi_{2}, \eta_{1}\right.$, $\eta_{2}$ ) with fixed $(\mathrm{x}, \mathrm{y})$ is approximated by the following sum over grid cells: 


$$
\begin{aligned}
& \frac{1}{2 \pi} \iint_{A} \frac{\left(x-x^{\prime}\right) \pm \imath\left(y-y^{\prime}\right)}{\left(\left(x-x^{\prime}\right)^{2}+\left(y-y^{\prime}\right)^{2}\right)^{3 / 2}} B_{z}\left(x^{\prime}, y^{\prime}\right) d x^{\prime} d y^{\prime} \\
& =\sum_{i=1}^{N} \frac{B_{z}\left(x^{\prime}, y^{\prime}\right)}{2 \pi}\left[\int_{\eta_{1}}^{\eta_{2}} \int_{\xi_{1}}^{\xi_{2}} \frac{\xi \pm \imath \eta}{\left(\xi^{2}+\eta^{2}\right)^{3 / 2}} d \xi d \eta\right] \Delta_{x} \Delta_{y}
\end{aligned}
$$

where we have the variables $\xi_{1},=x-x_{1}{ }_{1}, \xi_{2},=x-x_{2}{ }_{2}, \quad \eta_{1}=y-y_{1}{ }_{1}, \quad \eta_{2}=y-y_{2}{ }_{2}$ and $\Delta_{x} \Delta_{y}$ is an element of area given by $\left(\xi_{1}-\xi_{2}\right)\left(\eta_{1}-\eta_{2}\right)$. The integrals can be evaluated (cf. J. W. Harvey 1966) and they become ${ }^{3}$ :

$$
K\left(x, y, x_{i}{ }^{\prime}, y_{i}{ }^{\prime}\right)=\frac{1}{2 \pi}\left[\begin{array}{c}
\ln \frac{\left(\eta_{2}+\left(\eta_{2}{ }^{2}+\xi_{1}{ }^{2}\right)^{1 / 2}\right)\left(\eta_{1}+\left(\eta_{1}{ }^{2}+\xi_{2}{ }^{2}\right)^{1 / 2}\right)}{\left(\eta_{2}+\left(\eta_{2}{ }^{2}+\xi_{2}{ }^{2}\right)^{1 / 2}\right)\left(\eta_{1}+\left(\eta_{1}{ }^{2}+\xi_{1}{ }^{2}\right)^{1 / 2}\right)}+ \\
l \ln \frac{\left(\xi_{2}+\left(\eta_{1}{ }^{2}+\xi_{2}{ }^{2}\right)^{1 / 2}\right)\left(\xi_{1}+\left(\eta_{2}{ }^{2}+\xi_{1}{ }^{2}\right)^{1 / 2}\right)}{\left(\xi_{2}+\left(\eta_{2}{ }^{2}+\xi_{2}{ }^{2}\right)^{1 / 2}\right)\left(\xi_{1}+\left(\eta_{1}{ }^{2}+\xi_{1}{ }^{1 / 2}\right)\right.}
\end{array} \Delta_{x} \Delta_{y}\right.
$$

For the case $\eta=\xi=0$ the logarithmic expression for the integral in $M$ is non-singular with the intergral being zero for the cell centered on $(\xi, \eta)=(0,0)$. Below we can consider either the real or imaginary part of the equation, and there by recover Bz exterior data separately by using either the Bx or the By interior data.

Now we can evaluate the Fredholm integral equation using Singular Value Decomposition (SVD) regularization techniques. We have the linear system,

$$
\sum_{i=1}^{N} \bar{B}_{z}\left(x_{i},{ }^{\prime}, y_{i}{ }^{\prime}\right) K\left(x, y_{j}, x_{i}{ }_{i}, y_{i}{ }^{\prime}\right)=F\left(x_{i}, y_{i}\right), \quad 1 \leq j \leq N
$$

where $\mathrm{x}_{\mathrm{i}}{ }^{\prime}, \mathrm{y}_{\mathrm{i}}{ }^{\prime} \in I M$ and $\mathrm{x}_{\mathrm{j}}, \mathrm{y}_{\mathrm{j}} \in M$, or in operational notation we have

$$
\mathbf{K} \overline{\mathbf{B}}_{\mathbf{z}}=\mathbf{F}
$$

3 The equations can be derived using Gradshteyn and Ryzhik (1965) equations 2.264.6 (p.83) and 2.271.4 (p. 86). Note, if we define the center of a square cell to be $\left(\mathrm{x}_{\mathrm{i}}, \mathrm{y}_{\mathrm{i}}\right)$ and $\mathrm{x}_{1}{ }^{\prime}=\mathrm{x}_{\mathrm{i}}-\Delta / 2, \mathrm{x}_{2}{ }^{\prime}=\mathrm{x}_{\mathrm{i}}+\Delta / 2, \mathrm{y}_{1}{ }^{\prime}{ }^{\prime}=\mathrm{y}_{\mathrm{i}}-\Delta / 2$, and $\mathrm{y}_{2}{ }^{\prime}=\mathrm{y}_{\mathrm{i}}+\Delta / 2$, then we have that $\xi_{1,2}=\xi \pm \Delta / 2$ and $\eta_{1,2}=\eta \pm \Delta / 2$. Furthermore $K\left(x, y, x_{i}{ }^{\prime}, y_{i}{ }^{\prime}\right)=K(\xi, \eta)$. 
This system of unknowns $\overline{\mathbf{B}}_{\mathrm{z}}\left(\mathrm{x}_{\mathrm{i}}{ }^{\prime}, \mathrm{y}_{\mathrm{i}}^{\prime}\right)$ can be ill-posed by not having a uniquely determined solution or the solution can not depend continuously on the data contained in $\mathbf{F}\left(\mathrm{x}_{\mathrm{i}}, \mathrm{y}_{\mathrm{j}}\right)$. Using a Tikhonov-Phillips filter in the SVD formulation, we can stabilize the approximation. This filter solves the Tikhonov regularization problem of minimizing

$$
\left\|K \bar{B}_{z}-F\right\|^{2}+\gamma^{2}\left\|\bar{B}_{z}\right\|^{2}
$$

where $\gamma$ is a called the regularization parameter, and it is a solution of

$$
\left(\mathbf{K}^{*} \mathbf{K}+\gamma^{2} \mathbf{I}\right) \overline{\mathbf{B}}_{\mathbf{z}}=\mathbf{K}^{*} \mathbf{F}
$$

(Kress 1989, Natterer 1986). The regularization parameter introduces a smoothness condition on the solution of $\bar{B}_{z}$.

The SVD of $\mathbf{K}$ gives $\mathbf{K}$ in the form

$$
\mathbf{K}=\mathbf{U W} \mathbf{V}^{*}
$$

where $*$ indicates the transpose of the matrix, $\mathbf{U}$ and $\mathbf{V}$ are orthonormal, i.e.

$$
\begin{array}{ll}
\sum_{i=1}^{N} U_{i k} U_{i n}=\delta_{k n} & \text { for } 1 \leq \mathrm{k} \leq \mathrm{N}, 1 \leq \mathrm{n} \leq \mathrm{N} \\
\sum_{i=1}^{N} V_{i k} V_{i n}=\delta_{k n} & \text { for } 1 \leq \mathrm{k} \leq \mathrm{N}, 1 \leq \mathrm{n} \leq \mathrm{N}
\end{array}
$$

and $\mathbf{W}$ is diagonal with diagonal elements $\mathrm{w}_{\mathrm{k}}$, i, i.e. the singular values of $\mathbf{K}$, (Press, Flannery, Teukolsky, and Vetterling 1986, section 2.9, p. 53). Then the generalize inverse is given by

$$
\mathbf{K}^{-1}=\mathbf{V} \mathbf{W}^{-1} \mathbf{U}^{*}
$$

where instabilities occur for small $\mathrm{w}_{\mathrm{i}}$ 's in the formal solution

$$
\left[\overline{\mathbf{B}}_{\mathbf{z}}\right]_{\mathrm{i}}=\left[\mathbf{K}^{-1} \mathbf{F}\right]_{\mathbf{i}}=\left[\left(\mathbf{V} \mathbf{W}^{-1} \mathbf{U}^{*}\right) \mathbf{F}\right]_{\mathrm{i}}=\sum_{k=1}^{N} w_{k}^{-1} \sum_{j=1}^{N} U_{j k} F_{j} V_{i j} .
$$

The filter smooths out the singular functions assoicated with the small singular values, i.e., the high frequency components (Craig and Brown 1986, Natterer 1986). Using the Tikhonov-Phillips filter, $1 /\left(1+\left(\gamma / \mathrm{w}_{\mathrm{k}}\right)^{2}\right.$, we have the regularized (stable) solution

$$
\left[\overline{\mathbf{B}}_{z}\right]_{i}=\sum_{k=1}^{N} \frac{w_{k}}{w_{k}^{2}+\gamma^{2}} \sum_{j=1}^{N} U_{j k} F_{j} V_{i j} .
$$

The effect of the magnetic flux outside of the magnetogram can now be accounted for within the context of the regularized solution of the inverse matrix equation. The regularization process provides a stable algorithm for constructing an approximate solution which accounts for numerical and stochastic errors.

\section{Application}

\subsection{A SPECIFIC NUMERICAL EXAMPLE.}

In this section, a specific numerical application will be given. First, however, we will consider the interior Schmidt inversion problem, i.e., the problem above except that one of the transverse components of the magnetic field is given within the field of view and the longitudinal field is derived. Then, we will consider the exterior Schmidt inversion problem in which an area of exactly the same size adjacent to the 
magnetogram contains the flux to be resolved. The magnetogram lies in the region: $-10 \leq(\mathrm{x}, \mathrm{y}) \leq 10$. The elemental cell size are $2 \times 2$ in each case, which give an array size of $11 \times 11$ for $\mathrm{Bx}$ or with a column vector of 121 elements. Hence the K-matrix is a $121 \times 121$ matrix for these numerical examples. The normal fluxes will be defined by pistion functions, i.e., by a radii and a constant values within these radii. The configuration will be the same as in Figure 2.

In terms of the first numerical application, lets consider the interior Schmidt inversion problem . The problem is still ill-posed but the conditions number $\left(\operatorname{cond}(\mathrm{K})=\|\mathrm{K}\|\left\|\mathrm{K}^{-1}\right\|\right)$ for the associate matrix is smaller since the matrix elements of $\mathrm{K}$ decrease like $\approx(\Delta / \max (\xi))^{2}$, as $\quad \xi=\mathrm{x}-\mathrm{x}$ ' (or $\eta=\mathrm{y}-\mathrm{y}^{\prime}$ ) increases, and for the exterior region the distance are larger. If cond $(\mathrm{K})$ is not too large, the problem is said to be well-conditioned and the solution is stable with respect to measurement noise; otherwise, the solution is ill-conditioned (Bertero 1986, Press et al. 1986). In both the interior and exterior problem the matrix $\mathrm{K}$ is singular, but in the interior problem, the rank of $\mathrm{K}$ is larger. This is why we consider this stable solution first, but we also consider it since it is heuristic easier to concieve.

In Figure 3, we show the results of the numerical solution of the interior Schmidt inversion problem. In the top panel (A, B, C) the actual field components are shown (Bz, By, Bz). In the second row of panels $(\mathrm{D}, \mathrm{E}, \mathrm{F})$ the inverted values of $\mathrm{Bz}$ is given using $\mathrm{Bx}$ and $\gamma^{\prime} \mathrm{s}$ of $10^{-8}, 10^{-4}$, and $10^{-2}$.

In Figure 4, the Green's function for a specific field coordinates are shown for both $\mathrm{x}$ and $\mathrm{y}$-components. The asymmetric nature of the functions are clearly seen. The K-matrix is a collection of these fucntions off set by the specific field coordiantes.

In Figure 5, we show the results of the numerical solution of the exterior Schmidt inversion problem. In the top panel (A, B, C) the actual field components are shown (Bz, By, Bz). In the second (third) row of panels (D, E, F) the inverted values of $\mathrm{Bz}$ is given using $\mathrm{Bx}(\mathrm{By})$ and $\gamma^{\prime} \mathrm{s}$ of $10^{-8}, 10^{-4}$, and $10^{-2}$. In this figure the exterior field is composed of two symmetric magnetic fields situtated above the field of view of the magnetogram. An equivalent noise levels of $10 \mathrm{G}$ is introduce. In the "observed" Bx and By components.

In Figure 6, we show the results of the numerical solution of the exterior Schmidt inversion problem. In the top panel (A, B, C) the actual field components are shown (Bz, By, Bz). In the second (third) row of panels (D, E, F) the inverted values of $\mathrm{Bz}$ is given using $\mathrm{Bx}(\mathrm{By})$ and $\gamma^{\prime} \mathrm{s}$ of $10^{-8}, 10^{-4}$, and $10^{-2}$. In this figure the exterior field is composed of two offset magnetic fields situtated above the field of view of the magnetogram. An equivalent noise levels of $0.1 \mathrm{G}$ is introduce. In the "observed" Bx and By components. The domination of the near exterior field leads to a solutions dominated by that "sunspots".

\subsection{METHOD OF ESTIMATING THE REGULARIZATION PARAMETER}

The regularization parameter stabilizes the inversion process by filtering out the singular functions. The "solutions" of the regularization mehtods depends on the selection of the appropriate regularization parameter, i.e. filter. Tikhonov and Arsenin (1977) show the connection of optimizing the regularization parameter with the Wiener filter, i.e. the mean least square filter. Hence the determination of the regularization can be determined by statistical arguments. This argument uses extra information to stabilize the method and uses Baysian strategies by involking a priori probability distribution for the solution (Craig and Brown 1986, Turchin et al. 1971). Such arguments use the size of the errors. An alternative approach, that uses the actual errors, instead of the assumed error distributions, is the generalized cross-validation method (Bates and Wahba 1982, and Bertero 1986). The idea is to use the data points themselves to predict a "good" value of the regularization parameter by being able to predict the missing data points. This is seen that form the Tikhonov-Phillips formulation that we can write down the following formulae that predicts the value of $\mathrm{Bx}$ from the intial input via the solution of $\mathrm{Bz}$ :

$$
\mathbf{B}^{\prime}{ }_{x}=\mathbf{K}^{-1} \quad \overline{\mathbf{B}}_{\mathbf{z}}=\mathbf{K}^{-1} \mathbf{K}^{*}\left(\mathbf{K}^{*} \mathbf{K}+\gamma^{2} \mathbf{I}\right)^{-1} \mathbf{B}_{x} .
$$


Then minimizing the norm $\left\|\mathrm{B}^{\prime}{ }_{\mathrm{x}}-\mathrm{B}_{\mathrm{x}}\right\|^{2}$ leads to the generalized cross-validation. Golub et al. (1979) show that the genralized cross-validation formula $\mathrm{V}(\gamma)$ can be approximated in the following form which is computational faster:

$$
V(\gamma)=\left(\frac{1}{N^{2}} \operatorname{Tr}[I-\widetilde{A}(\gamma)]\right)^{-2}\left(\frac{1}{N^{2}}\left\|[I-\tilde{A}(\gamma)] B_{x}\right\|\right)^{2},
$$

where

$$
\tilde{A}(\gamma)=\frac{K K^{*}}{K K^{*}+\gamma^{2} I},
$$

and $\mathrm{N}^{2}$ is number of elements in the array. The generalized cross-validation is plotted in figure 7. For small noise a local minimum is assured. (Tikhonov and Arsenin 1977,p. 165).

Since the basic effect of the regularization parameter $\gamma$ is to effectively smooth the solution, we can also select $\gamma$ by choosing the solution which "best" matches the smoothness of the magnetic field normal component in the observed field of view. This assumes a priori knowledge of the equal likelyhood of the two fields being of equal smoothness. However, we are accuractually better off than this simple assumption since we are using the solution to solve of the potential field in the interior. Therefore the effects of the solution is smoothed out further by the integration distance for the exterior field. In figure 7 we plot the results of the various figure of merits to determine the regularization parameter and the actual error. The two smoothness criteria are $\left\|\nabla B_{z}\right\|^{2}$ and $\left|\partial^{2} B_{x} / \partial x^{2}+\partial^{2} B_{y} / \partial y^{2}\right|^{2}$. All the criteria give the same value for the regularization parameter. These methods look for solutions with small derivatives and hence impose smoothness, i.e. the avoid large amplitude variations which made the initial problem ill-posed by removing the high frequency characteristics of the problem. The ill-poseness of the problem has been corrected by a priori knowledge of the nature of the solution.

\subsection{GENERALIZING THE TECHNIQUES}

The generalization of the problem is to specify the geometric configuration of the grid outside of the magnetogram without a priori knowledge of the position of the exterior points. Assuming that within the magnetogram we have a $\mathrm{N}$ by $\mathrm{N}$ grid of cells, then outside of the magnetogram we have to have a collection of $\mathrm{N}$ by $\mathrm{N}$ grid of cells surrounding the magnetogram in order to keep the SVD matrices squared, for simplicity. If the area of the grids outside of the magnetogram, i.e. in $\Gamma M$, is the same area as magnetogram, $\Delta^{2}$, then the number of rows and columns that are added, $\mathrm{m}$, around the magnetogram to have the same number of grid cells outside equaling to the number inside is given by

$$
m=\frac{\sqrt{2}-1}{2} \text { or } m=\frac{N^{\prime} \Delta-N \Delta}{2 \Delta},
$$

where $N^{\prime} \Delta=\sqrt{ } 2 N \Delta$ and $N^{\prime} \Delta$ is the outside dimension of the extended area; $N^{2}$ is the number of cells in the magnetogram. Then $m / N$ is the ratio of the linear increase of the extended bounds on the magnetogram.

There is no reason we could not consider the grid cells outside of the magnetogram to be larger. For large grid cells in $I \mathrm{M}$, we can take the linear increase of the extend bounds to be 3 , and the new grid cell linear dimension to by $N / n$, where we have $N\left(N^{2} / n^{2}+1\right)^{1 / 2}=3 N$. (For numerical evaluation we wish to have $N / n$ an integer.) Hence we have the extended region to eight times the area of the magnetogram when we take exterior grids be about three times larger, actually $2 \sqrt{2}$. Because of the square of 2 , the actual grid pattern will necessary be modified, giving some rough outer edges, in order to keep the square matrix and square grid configurations. The mathematical algorithm for the generalization is the same as given above.

\section{Conclusion}


In the classical potential field extrapolation process from a planar boundary, we need only the normal component over the entire plane wiht the additional assumption that the net flux in zero. If we have data from only a finite field of view then the basic assumption is that the flux outside the viewing area in zero. We have considered in the text the solution of the potential extrapolation problem using the three components of the observed magnetic field to extrapolate the field. We have effectively thaken the three components of the observed region and extrapolated the field. This is the same problem posed by the Cauchy problem for extrapolating the magnetic field ( Wu, et al. 1990, Gary and Musielak 1992). In each case the field in ill-posed and regularization techniques must be imposed. Here we have shown a potential solution which can be used to solve a specific problem of a finite field of view magnetogram being influence by flux outside of the field of view. Futhermore, the Cauchy solution does not blowup as a function of height. However, we have not investigated the error as a function of height.

\section{Acknowledgements}

Part of this work was supported by the NASA Office of Solar Physics. We thank Rainer Kress for his helpful comments and discussions.

\section{Appendix A. Extended Biot-Savart Law}

This appendix derives the generalized Biot-Savart law given by equation (3) in the text. Starting from the Gauss' ivergence theorem $\int \nabla \cdot \mathbf{A} \quad \mathrm{dv}=\int \mathbf{A} \cdot \mathbf{n}$ da and letting $\mathbf{A}=\phi \nabla \psi$ and using the idenitity $\nabla \cdot \mathbf{A}=\nabla \cdot(\phi \nabla \psi)=\phi \nabla^{2} \psi+\nabla \phi \cdot \nabla \psi$, we have $\int\left(\phi \nabla^{2} \psi+\nabla \phi \cdot \nabla \psi\right) \mathrm{dv}=\int \phi \nabla \psi \cdot \mathbf{n} \mathrm{ds}=\int \phi \partial \psi / \partial$ n. Now interchanging $\phi$ and $\psi$ and subtracting the two equations, have Green's second identity or Green's theorem:

$$
\int_{v}\left(\phi \nabla^{2} \psi-\psi \nabla \phi\right) d v=\oint_{s}\left(\phi \frac{\partial \psi}{\partial n}-\psi \frac{\partial \phi}{\partial n}\right) d a
$$

which relates a volume integral to the a surface integral about the enclose the volume. If we let $\phi=B_{x}$, $B_{x}$, and $B_{x}$ and let $\psi=G\left(\mathbf{x}, \mathbf{x}^{\prime}\right)$, and sum the three components, we have a vector form of the Green's theorem:

$$
\int_{v}\left(\mathbf{B} \nabla^{2} G-G \nabla^{2} \mathbf{B}\right) d v=\oint_{s} \mathbf{B}(\mathbf{n} \cdot \nabla G)-G(\mathbf{n} \cdot \nabla) \mathbf{B} d a,
$$

( cf. Jackson 1963, eqn. 9.68 p. 283). The function is the Green's function which is choosen such that

$$
\nabla^{2} G\left(x, x^{\prime}\right)=-4 \pi \delta(r),
$$

then allows the magnetic field intensity to be written as the following

$$
B(x)=-\frac{1}{4 \pi} \int_{v} G \nabla^{2} \mathbf{B} d v-\frac{1}{4 \pi} \oint_{s} \mathbf{B}(\mathbf{n} \cdot \nabla G)-G(\mathbf{n} \cdot \nabla) \mathbf{B} d a
$$

There are three special choices for $\mathrm{G}\left(\mathbf{x}, \mathbf{x}^{\prime}\right)$ for the upper half-plane case with a planar boundary at $\mathrm{z}=0$; the choices are (a) $G\left(\mathbf{x}, \mathbf{x}^{\prime}\right)=1 /\left|x-x^{\prime}\right|$, (b) $G\left(\mathbf{x}, \mathbf{x}^{\prime}\right)=1 /\left|\mathbf{x}-\mathbf{x}^{\prime}\right|-1 /\left|\mathbf{x}-\mathbf{x}^{*}\right|$, or (c) $G\left(\mathbf{x}, \mathbf{x}^{\prime}\right)=1 /\left|\mathbf{x}-\mathbf{x}^{\prime}\right|+1 /\left|\mathbf{x}-\mathbf{x}^{*}\right|$, where $\mathbf{x}=(\mathrm{x}, \mathrm{y}, \mathrm{z})$ are the field points, $\mathbf{x}^{\prime}=\left(\mathrm{x}^{\prime}, \mathrm{y}^{\prime}, \mathrm{z}^{\prime}\right)$ are the source points and $\mathbf{x}^{*}=\left(\mathrm{x}^{\prime}, \mathrm{y}^{\prime},-\mathrm{z}^{\prime}\right)$ are the image points. The case (a) leads to the formulation that we consider in more details. In case (b) we have $G\left(\mathbf{x}, \mathbf{x}^{\prime}\right)=0$ on the surface, i.e. the harmonic Dirichlet Green's functions for the half-plane ,and case (c) we 
have $\nabla \mathrm{G}\left(\mathbf{x}, \mathbf{x}^{\prime}\right)=0$ on the surface, i.e., the harmonic Neumann Green's functions for the half-plane (Duff and Naylor 1966, p. 272). For a potential field, Case (b) can be shown to lead to the same set of equations ${ }^{4}$ which are derived from considering $\nabla \times \nabla \times \mathbf{B}=\nabla^{2} \mathbf{B}=0$ for the case $\mathrm{J}=0$.

Now consider the integrand

$$
[]=\mathbf{B}(\mathbf{n} \cdot \nabla G)-G(\mathbf{n} \cdot \nabla) \mathbf{B}
$$

and using the identities (a) $\mathbf{n} \times(\mathbf{B} \times \nabla G)=\mathbf{B}(\mathbf{n} \cdot \nabla \mathrm{G})-(\mathbf{n} \cdot \mathrm{B}) \nabla \mathrm{G}$; (b) $\nabla \mathrm{G} \times(\mathbf{n} \times \mathbf{B})=\mathbf{n}(\mathbf{B} \cdot \nabla \mathrm{G})-\mathrm{B}(\mathbf{n} \cdot \nabla \mathrm{G})$; (c) $(\mathrm{n} \cdot \nabla)(\mathrm{GB})=\mathbf{B}(\mathbf{n} \cdot \nabla \mathrm{G})+\mathrm{G}(\mathbf{n} \cdot \nabla) \mathrm{B}$; we have

$$
\begin{gathered}
{[]=(\mathbf{n} \cdot \nabla)(G \mathbf{B})-2 \mathbf{B}(\mathbf{n} \cdot \nabla G)} \\
{[]=(\mathbf{n} \cdot \nabla)(G \mathbf{B})-\mathbf{n} \times(\mathbf{B} \times \nabla G)-(\mathbf{n} \cdot \mathbf{B}) \nabla G-\mathbf{n}(\mathbf{B} \cdot \nabla G)+\nabla G \times(\mathbf{n} \times \mathbf{B})} \\
{[]=(\mathbf{n} \cdot \nabla)(G \mathbf{B})-\mathbf{n} \times(\mathbf{B} \times \nabla G)-\mathbf{n}(\mathbf{B} \cdot \nabla G)-(\mathbf{n} \cdot \mathbf{B}) \nabla G-(\mathbf{n} \times \mathbf{B}) \times \nabla G}
\end{gathered}
$$

Now using the identities (d) $\nabla \times(\mathrm{GB})=\mathrm{G}(\nabla \times \mathbf{B})+\mathbf{B} \times \nabla \mathrm{G}$ and (e) $\nabla \cdot(\mathbf{B G})=\mathbf{B} \cdot \nabla \mathrm{G}+\mathrm{G} \nabla \cdot \mathbf{B}$, we have

$$
[]=(\mathbf{n} \cdot \nabla)(G \mathbf{B})+\mathbf{n} \times \nabla \times(G \mathbf{B})-\nabla \cdot(G \mathbf{B})-(\mathbf{n} \cdot \mathbf{B}) \nabla G-(\mathbf{n} \times \mathbf{B}) \times \nabla G-G \mathbf{n} \times(\nabla \times \mathbf{B})
$$

By using the identities ${ }^{5}$ (f) $\int \mathbf{n} \cdot \mathbf{A} \mathrm{da}=\int \nabla \cdot \mathbf{A} \mathrm{dv} ;(\mathrm{g}) \int \mathbf{n} \times \mathbf{A} \mathrm{da}=\int \nabla \times \mathbf{A} \mathrm{dv} ;$ (f) $\int \phi \mathbf{n} \mathrm{da}=\int \nabla \phi \mathrm{dv}$ where the surface integrals enclose the volume, the first three terms on the [ ] when integrated of the volume gives zero, since $\nabla \times \nabla \times(\mathrm{GB})=\nabla(\nabla \cdot(\mathrm{GB}))-\nabla^{2}(\mathrm{~GB})$. Hence we

$$
B(x)=-\frac{1}{4 \pi} \int_{v} G \nabla^{2} \mathbf{B} d v-\frac{1}{4 \pi} \oint_{s}\left[-(\mathbf{n} \cdot \mathbf{B}) \nabla^{\prime} G-(\mathbf{n} \times \mathbf{B}) \times \nabla^{\prime} G-G \mathbf{n} \times\left(\nabla^{\prime} \times \mathbf{B}\right)\right] d a^{\prime}
$$

The second term of the surface integral can be written using, first $\nabla \times \mathbf{B}=\mu \mathbf{J}$, and then identity (g) above, and final using $\nabla \times \mu \mathbf{J}=\nabla \times \nabla \times \mathbf{B}=\nabla(\nabla \cdot \mathrm{B})+\nabla^{2} \mathrm{~B}$,

$$
\oint_{S}\left[-G \mathbf{n} \times\left(\nabla^{\prime} \times \mathbf{B}\right)\right] d a^{\prime}=\int_{v}(-\nabla G \times \mu \mathbf{J}-G(\nabla \times \mu \mathbf{J})) d v=\int_{v}\left(-\nabla G \times \mu \mathbf{J}-G \nabla^{2} \mathbf{B}\right) d v
$$

${ }^{4}$ If $\mathrm{G}=0$ on the surface then we have

and expanding

$$
B(x)=-\frac{1}{4 \pi} \int_{v} G \nabla^{2} \mathbf{B} d v-\frac{1}{4 \pi} \oint_{s} \mathbf{B}(\mathbf{n} \cdot \nabla G) d a
$$

$$
-\frac{1}{4 \pi} \int_{v} G \nabla^{2} \mathbf{B} d v=-\frac{1}{4 \pi} \int_{v} G(n \times(\nabla \times B)) d a-\frac{\mu}{4 \pi} \int_{v} \nabla G \times J d v
$$

and we have the magnetic field intensity in terms of the volume current density and the component of $B$ on the surface

$$
B(x)=-\frac{\mu}{4 \pi} \int_{v} \nabla G \times J d v-\frac{1}{4 \pi} \oint_{s} \mathbf{B}(\mathbf{n} \cdot \nabla G) d a
$$

For $\mathrm{G}\left(\mathbf{x}, \mathbf{x}^{\prime}\right)=1 / \mathrm{r}-1 / \mathrm{r}^{*}$, we have $\nabla \mathrm{G}=2 \mathrm{z} \mathbf{k} / \mathrm{r}$. The volume integral represents the magnetic field from the volume currents and their image currents. Redefining the variable the formulation can be seen to that dicussed by Hagyard, Low, and TandbergHanssen 1981.

A similar equation is obtained if $\nabla \mathrm{G}=0$ on the surface; starting with

$$
B(x)=-\frac{1}{4 \pi} \int_{v} G \nabla^{2} \mathbf{B} d v-\frac{1}{4 \pi} \oint_{s}-G(\mathbf{n} \cdot \nabla) \mathbf{B} d a
$$

and expanding the volume integral as above and using the identities c, d, and e above. However, the Green's function have different definitions/.

${ }^{5}$ These formulae do not apply at $\mathrm{x}=\mathrm{x}$ ', but if the singularity is excluded by taking the appropriate surfaces, the contribution of the region around the singularity gives a zero contribution in the limit as the surface closes around the singularity (Jackson 1963, p. 284). 
Therefore

$$
B(x)=-\frac{1}{4 \pi} \int_{v} \mathbf{J} \times \nabla G d v-\frac{1}{4 \pi} \oint_{s}\left[(\mathbf{n} \cdot \mathbf{B}) \nabla^{\prime} G-(\mathbf{n} \times \mathbf{B}) \times \nabla^{\prime} G\right] d a^{\prime}
$$

or for $\mathrm{G}=1 / \mathrm{r}=1 /\left|\mathbf{x}-\mathbf{x}^{\prime}\right|$ we have

$$
B(x)=-\frac{1}{4 \pi} \int_{v} \mathbf{J} \times \nabla\left(\frac{1}{r}\right) d v-\frac{1}{4 \pi} \oint_{s}(\mathbf{n} \cdot \mathbf{B}) \nabla^{\prime}\left(\frac{1}{r}\right) d a^{\prime}-\frac{1}{4 \pi} \oint_{s}(\mathbf{n} \times \mathbf{B}) \times \nabla^{\prime}\left(\frac{1}{r}\right) d a^{\prime}
$$

The first surface integral is the double layer configuration with the monopole surface charge configuration. The second surface integral is the surface current contribution. The two integrals are identical.

\section{Appendix B. Schmidt Method and the Green FuntionOperator}

The formulation given in the text was employed to test the numerical codes. It is important to note that the matrix operator $\mathrm{K}$ in the equation $\mathrm{KxBz}=\mathrm{Bx}$ and $\mathrm{KyBz}=\mathrm{Bx}$ are similar and the nature of the matrix is seen the Figure B1 below.

\section{References}

Adams, J. , and Pneuman, G. W.: 1976, Solar Phys. 46, 185

Altschuler, M. D., and Newkirk, G. Jr.: 1969, Solar Phys. 9, 131

Alissandrakis, C. E.: 1982, Astro. Ap. 100, 97

Bates, D. M., Wahba, G.:1982, in C. T. H. Baker and G. F. Miller (eds.), Treatment of Integral Equations by Numerical Methods, Academic Press, New York, p.283.

Bertero, M.: 1986, in G. Talenti (ed.), Inverse Problems, Springer-Verlag, New York, p. 52.

Chui, Y. T., and Hilton, H. H.: 1977, Astrophys. J. 212, 873

Craig, I. J. D., and Brown, J. C.: 1986, Inverse Problems in Astronomy, Adams Hilger, Boston

Duff, G. F. D., and Naylor, D.: 1966, Differential Equations of Applied Mathematics, Wiley, New York, p. 272

Gary, G. A., Moore, R. L., Hagyard, M. J., and Haisch, B. M.: 1987, Astrophys, J. 314, 728

Gary, G. A., and Musielak, Z. E.: 1992, Astrophys, J. 392, 722

Glasko, V. B.: 1984, Inverse Problems of Mathematical Physics, American Institute of Physics, New York

Golub, G. H., Heath, M., Wahba, G.: 1979, Technometrics 21, 215 
Gradshteyn , I. S., and Rhyzhik, I. M.: 1965, Table of Integrals, Series, and Products, Academic Press, New York

Groetsch, C. W.: 1984, The Theory of Tikhonov Regularization for Fredholm Equations of the First Kind, Pitman, Boston

Hagyard, M. J., Low, B. C., and Tandberg-Hanssen, E.: 1981, Solar Phys., 73, 257

Harvey, J.W. Evaluation of Solar Magnetograms, Astro-Geophysical Memorandum No. 172, High Altitude Observatory, Boulder, Colorado, 9 May 1966

Jackson, J. D.: 1963, Classical Electrodynamics, John Wiley, New York.

Kress, R.: 1987, "On an Ill-Posed Problem for Constant Alpha Force-Free Fields", in H. W. Engle and C. W. Groetsch (eds.), Inverse and Ill-Posed Problems, Academic Press, New York, p. 417

Kress, R.: 1989, “Linear Integral Equations”, Springer-Verlag, New York, p. 238

Levine, R. H.: 1982, Solar Phys. 79, 203

Mikic, et al. 1985

Mikic, Z., Barnes, D. C., and Schnack, D. D.: 1988, Astrophys. J., 328, 830

Mikic, et al. 1989

Morozov, V. A.: 1993, “Regularization Methods for Ill-Posed Problems”, CRC Press, Boca Raton

Natterer, F.: 1986, \{lit Numerical Treatment of Ill-posed Problems\}, in “Inverse Problems", ed. R. Talenti, Springer-Verlag, New York ,p. 143

Panofsky, W. H., and Phillips, M.: 1962, Classical Electricity and Magnetism, Addison-Wesley, New York

Poletto, G., and Kopp, R. A.:1988, Solar Phys. 116, 163

Press, W. H, Flannery, B. P., Teukolsky, S. A. and W. T. Vetterling, W. T.: 1986 “Numerical Recipes”, Cambridge University Press, New York, section 2.9, p. 53.

Riesebieter, W., and Neubauer, F. M.: 1979, Solar Phys. 63, 127

Sakurai, T., 1980, Solar Phys. 76, 301 
Schatten, K. H., Wilcox, J. M., and Ness, N. F|.: 1969, Solar Phys. 6, 442

Semel, M.: 1967, Ann. Astrophys. 30, 513

J. A. Stratton, Electromagnetic Theory, (New York: McGraw-Hill), 1941, p. 250-254., sec. 4.14

Schmidt, H. U.:1963, in W. N. Hess, `The Physics of Solar Flares”, NASA SP-50, Washington, D. C., 1963, p. $107-116$

Teuber, D., Tandberg-Hanssen, E., and Hagyard, M. J.: 1977, Solar Phys. 53, 97

Tikhonov, A. N., and Arsenin, V. Y.: 1977, 'Solutions of Ill-Posed Problems”, John Wiley and Sons, New York.

Turchin, V. F., Kozlov, V. P., and Malkevich, M. S.: 1971, Sov. Phys.-Usp. 13, 681

Wu, S. T, Chang, H. M., and Hagyard, M. J.: 1985, in M. J. Hagyard (ed.) in Measurements of Solar Vector Magnetic Fields, NASA CP-2374, p.17

Wu, S. T., Sun, M. T., Chang, H. M., Hagyard, M. J., and Gary, G. A.: 1990, Astrophys, J. 362, 698

This document was prepared using Microsoft Word Version 6.0a

File: C:ITEXTISVDPAP4.DOC

03/20/95 8:22 